Surfaces, Interfaces and Coatings Technology

Book Chapter

\title{
Effects of $\mathrm{O}_{2}$ Plasma Treatments on the Photolithographic Patterning of PEDOT: PSS
}

Deng-Yun Zheng ${ }^{1}$, Meng-Hsiang Chang ${ }^{1}$, Ci-Ling $\operatorname{Pan}^{1,2}$ and Masahito Oh-e $\mathrm{e}^{1 *}$

${ }^{1}$ Institute of Photonics Technologies, Department of Electrical Engineering, National Tsing Hua University, Taiwan

${ }^{2}$ Department of Physics, National Tsing Hua University, Taiwan

*Corresponding Author: Masahito Oh-e, Institute of Photonics Technologies, Department of Electrical Engineering, National Tsing Hua University, 101 Sec. 2 Kuang-Fu Road, Hsinchu 30013, Taiwan

Published April 21, 2021

This Book Chapter is a republication of an article published by Masahito Oh-e, et al. at Coatings in December 2020. (Zheng, D.Y.; Chang, M.-H.; Pan, C.-L.; Oh-e, M. Effects of $\mathrm{O}_{2}$ Plasma Treatments on the Photolithographic Patterning of PEDOT:PSS. Coatings 2021, 11, 31.

https://doi.org/10.3390/coatings11010031)

How to cite this book chapter: Deng-Yun Zheng, Meng-Hsiang Chang, Ci-Ling Pan, Masahito Oh-e. Effects of $\mathrm{O}_{2}$ Plasma Treatments on the Photolithographic Patterning of PEDOT:PSS. In: Alessio Bosio, editor. Surfaces, Interfaces and Coatings Technology. Hyderabad, India: Vide Leaf. 2021.

(C) The Author(s) 2021. This article is distributed under the terms of the Creative Commons Attribution 4.0 International License(http://creativecommons.org/licenses/by/4.0/), which permits unrestricted use, distribution, and reproduction in any medium, provided the original work is properly cited. 
Author Contributions: Conceptualization, C.-L.P. and M.O.; methodology, D.-Y.Z. and M.O.; validation, D.-Y.Z.; formal analysis, D.-Y.Z. and M.O.; investigation, D.-Y.Z. and M.-H.C.; data curation, D.-Y.Z.; writing - original draft preparation, D.Y.Z.; writing - review and editing, M.O.; visualization, M.O.; supervision, C.-L.P. and M.O.; project administration, C.-L.P. and M.O.; funding acquisition, C.-L.P. and M.O. All authors have read and agreed to the published version of the manuscript.

Funding: This work was supported by grants from the Ministry of Science and Technology (MOST), Taiwan, under Grant MOST 108-2221-E-007-094-MY2 as well as Grants MOST 1082622-M-007-006-CC1 and 109-2622-M-007-007-CC1 for industrial-academic collaborations jointly funded by MOST and Profound Material Technology Co., Ltd., in Taiwan.

Conflicts of Interest: The authors declare no conflict of interest.

\section{Abstract}

Poly(3,4-ethylenedioxythiophene) polystyrene sulfonate (PEDOT:PSS) is known for its potential to replace indium-tin oxide in various devices. Herein, when fabricating finger-type PEDOT:PSS electrodes using conventional photolithography, the cross-sectional profiles of the patterns are U-shaped instead of rectangular. The films initially suffer from non-uniformity and fragility as well as defects owing to undesirable patterns. Adding a small amount of hydrolyzed silane crosslinker to PEDOT:PSS suspensions increases the mechanical durability of PEDOT:PSS patterns while lifting off the photoresist. To further improve their microfabrication, we observe the effects of two additional oxygen $\left(\mathrm{O}_{2}\right)$ plasma treatments on conventional photolithography processes for patterning PEDOT:PSS, expecting to observe how $\mathrm{O}_{2}$ plasma increases the uniformity of the patterns and changes the thickness and U-shaped crosssectional profiles of the patterns. Appropriately exposing the patterns photoresist to $\mathrm{O}_{2}$ plasma before spin-coating PEDOT:PSS improves the wettability of its surface, including its sidewalls, and a similar treatment before lifting off the photoresist helps partially remove the spin-coated PEDOT:PSS 
that impedes the lift-off process. These two additional processes enable fabricating more uniform, defect-free PEDOT:PSS patterns. Both increasing the wettability of the photoresist patterns before spin-coating PEDOT:PSS and reducing its conformal coverage are key to improving the photolithographic microfabrication of PEDOT:PSS.

\section{Keywords}

Microfabrication; Photolithography; Patterning; PEDOT:PSS; Plasma; Thickness; Film; Surface; Height Profile; Terahertz Device

\section{Introduction}

Advancing terahertz $(\mathrm{THz})$ technology $[1,2]$ requires developing many functional quasi-optical components for $\mathrm{THz}$ devices such as phase shifters [3-8], filters [9], phase gratings [10], and polarizers [11]; however, most of these device components use indium-tin oxide (ITO) or metal electrodes, the transmittance of which is not necessarily high enough for $\mathrm{THz}$ devices.

To improve transmittance characteristics, on one hand, fingertype ITO electrodes [12], which mitigate the absorption of light via ITO, have been fabricated by patterning flat ITO electrodes. On the other hand, materials with higher transmittance in the $\mathrm{THz}$ region such as graphene [13] are actively being attempted to replace ITO. For this purpose, poly(3,4-ethylenedioxythiophene) polystyrene sulfonate (PEDOT:PSS) [14-19] is a promising candidate, and patterning PEDOT:PSS as a microfabrication strategy has been well studied. PEDOT:PSS exhibits high transmittance in the $\mathrm{THz}$ region, and because of its solutionprocess capability, it can be patterned using photolithography. Under these circumstances, replacing the finger-type ITO electrodes with patterned PEDOT:PSS ones would attain even higher transmittance in the $\mathrm{THz}$ region. Therefore, we attempted to fabricate finger-type electrodes using PEDOT:PSS; however, the films suffered from non-uniformity and fragility as well as defects owing to undesirable patterns. 
As wearable and portable electronics have continued to develop [20], PEDOT:PSS as a flexible transparent electrode has been gaining an important role in such optoelectronic devices [21]. For practical purposes, developing versatile patterning techniques is required for incorporating PEDOT:PSS into these optoelectronic devices. Micropatterning has been developed for organic materials, but some challenges have not yet been fully addressed; in particular, the surface morphology and electrical and optical properties of patterned PEDOT:PSS are adversely influenced by patterning processes. This is because organic materials, i.e., soft matter, that can be dissolved or dispersed in water or organic solvents are usually mechanically fragile and chemically sensitive. These difficulties have encouraged researchers to devise various approaches in different micro- and nanopatterning techniques to solve these problems and to ultimately achieve well-organized, uniformly patterned surfaces with fine structures.

In principle, photolithography is applicable to organic materials including PEDOT:PSS when the proper materials and processes are selected [16]. Traditional photolithography materials and processes can be used in the lift-off process. As another alternative method, using orthogonal solvents and corresponding photoresists enables creating submicrometer-scale PEDOT:PSS patterns $[22,23]$; however, they can potentially be damaged by aggressive organic solvents. In addition, PEDOT:PSS can be directly crosslinked by ultraviolet (UV) irradiation with an appropriate photoinitiator [24-26], but the resolution is limited to several micrometers. From the above-mentioned techniques, we choose traditional photolithography together with the lift-off process. In other words, we observe how PEDOT:PSS films can be patterned using traditional photolithography and how undesirable patterns or defects, if any, made by photolithographic processes can be altered and improved.

Plasma treatments are well known and offer many advantages in fabrication processes because they can modify the surface properties, but exposing a surface to plasma does not alter its bulk properties [27]. Plasma treatment can prime any surface for 
secondary manufacturing processes by eliminating all traces of contamination. Therefore, we are interested in observing the correlation between plasma treatments and the surface structures and morphologies of photolithographic PEDOT:PSS patterns while exploring ways to improve their quality.

In this study, we aim to tackle the aforementioned problems, i.e., the non-uniformity and fragility of the films as well as defects owing to undesirable patterns, and to improve the quality of photolithographic PEDOT:PSS patterns. Although photolithography has already been established for PEDOT:PSS, some material- and process-related factors seem to affect the quality of the films and patterns. For this purpose, we introduce two additional $\mathrm{O}_{2}$ plasma processes into traditional photolithography, and we analyze how each additional plasma treatment affects the PEDOT:PSS patterns, expecting to improve them by applying these two additional processes. From a practical perspective, exploiting these basic, unsophisticated fabrication processes while unveiling their effects helps further develop the photolithographic patterning of PEDOT:PSS thin films in a facile, familiar way.

\section{Materials and Methods}

\section{Materials and Procedure}

Soda-lime glass substrates with dimensions of $25 \times 25 \times 0.7 \mathrm{~mm}$ were sequentially sonicated in commercially available detergent RBS $^{\text {TM }} 35$ Concentrate (Thermo Fisher Scientific, Waltham, MA, USA and deionized water $(>18 \mathrm{M} \Omega \cdot \mathrm{cm})$ for $15 \mathrm{~min}$. To increase its hydrophilicity, the surface of the substrates was exposed to $\mathrm{O}_{2}$ plasma with a power of $100 \mathrm{~W}$ and $\mathrm{O}_{2}$ flowing at $15 \mathrm{sccm}$ for 3 min using a Samco-ucp PC-300 plasma cleaner (Ruggell, Liechtenstein). A positive photoresist AZ4620 (Merck KGaA, Darmstadt, Germany) was spin-coated onto the substrates at $4000 \mathrm{rpm}$ for $40 \mathrm{~s}$ and then baked at $90{ }^{\circ} \mathrm{C}$ for 7 min. The photoresist films were exposed to UV light for $7 \mathrm{~s}$ using an $\mathrm{EVG}^{\circledR} 620$ mask aligner. The photoresist films were developed in a developer solution AD-10 (Merck KGaA, Darmstadt, Germany) for $3 \mathrm{~min}$. 
Figure 1 illustrates the designed mask patterns of a unit cell together with an enlarged diagram of a corner of the electrode area showing a part of the finger-type electrodes near the edge. The total electrode dimensions are $14 \times 13 \mathrm{~mm}$, and each fingertype electrode is $20 \mu \mathrm{m}$ wide with a $20 \mu \mathrm{m}$ gap between neighboring electrodes. In practice, the four designed unit cells were laid out in a soda-lime mask with dimensions of $5 \times 5 \times$ 0.09 in $(12.7 \times 12.7 \times 0.229 \mathrm{~cm})$.

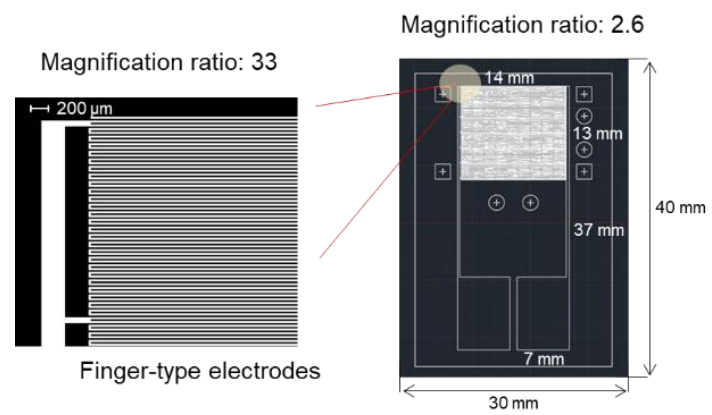

Figure 1: Designed mask pattern for a unit cell (right) and enlargement of the finger-type electrodes (left).

A PEDOT:PSS dispersion (PH1000, Heraeus Epurio Clevios ${ }^{\mathrm{TM}}$, Leverkusen, Germany) was filtered through a $0.45 \mu \mathrm{m}$ syringe filter and then mixed with 0.2 wt \% (3glycidyloxypropyl)trimethoxysilane (GOPS) [28,29], a hydrolyzed silane crosslinker (Sigma-Aldrich, St. Louis, MO, USA). The suspension was sonicated for $30 \mathrm{~min}$ to homogenize the PEDOT:PSS dispersion, which was then spin-coated at 3000 rpm for $60 \mathrm{~s}$ onto the patterned photoresist. The spin-coated substrates were baked at $130{ }^{\circ} \mathrm{C}$ for $15 \mathrm{~min}$ and finally sonicated in acetone to lift off the photoresist.

In our modified process, two $\mathrm{O}_{2}$ plasma treatments were independently added, one before spin-coating PEDOT:PSS and one before lifting off the photoresist. The $\mathrm{O}_{2}$ plasma power and the $\mathrm{O}_{2}$ flow rate were set to $100 \mathrm{~W}$ and $15 \mathrm{sccm}$, respectively, for both reaction ion etching (RIE) and plasma etching (PE) modes, and each operation time was varied. The latter mode is more isotropic and gentler, whereas in the former mode, the $\mathrm{O}_{2}$ species are generated more vertically and hit the substrates more vigorously because of the potential between the electrodes in the chamber. 
Surfaces, Interfaces and Coatings Technology

\section{Measurements}

A Dimension ICON scanning probe microscope system (Bruker, Billerica, MA, USA), which offers precisions of $<0.15 \AA \mathrm{nm}$ for $\mathrm{XY}$ noise and $<0.35 \AA \mathrm{Z}$ sensor noise, was used to measure the film thickness and probe its height profiles on the surface of the substrates. We prepared three samples for each set of conditions. For each sample, we measured at least three points in the upper, middle, and lower parts of the electrode area shown in Figure 1, and we measured each point three times. We also used a Quatek (Sheung Wan, Hong Kong) four-point probe test system, composed of a 5601TSR surface resistance tester and a QT-50 manual test console, to measure the surface resistance $R_{s}(\Omega)$ of films, from which the conductivity $\sigma(\mathrm{S} / \mathrm{cm})=1 /\left(R_{s} \cdot d\right)$ was deduced using the film thickness $d(\mathrm{~cm})$.

\section{Results}

\section{Transferring an Image to the Photoresist and the Fragility of PEDOT:PSS Patterns}

To draw a finger-type (stripe-type) image on the photoresist, a mask pattern (design drawing or pattern) is first transferred. The stripe-type-patterned photoresist works as a template or mold to create PEDOT:PSS patterns $[16,30]$. To this end, some process conditions are optimized using various quality engineering methods [31], i.e., by controlling the spin-coating rotational speed, baking temperature, UV-light-exposure time, and developing time, as described in the experimental section. Using these optimized conditions, we attain a high-quality patterned photoresist, and the patterns are transferred from the mask patterns with a gap of $20 \mu \mathrm{m}$ between neighboring patterns. By using the image (pattern) of the photoresist, PEDOT:PSS is then patterned via microprocessing.

Initially, the PEDOT:PSS patterns were very mechanically fragile, especially in the lift-off process, during which the substrates were shaken in an acetone bath. To remove the photoresist patterns from the substrates, they were inevitably shaken, which presumably applied a frictional force to the PEDOT:PSS patterns on the substrates. In fact, the PEDOT:PSS 
patterns were partially peeled off and bent out of the substrates, as shown in Figure 2. Simply immersing substrates in acetone, however, does not remove photoresist patterns from substrates; therefore, to avoid damaging the patterns, the films had to be strengthened. This fragility can be attributed to the weak anchoring of the PEDOT:PSS films to the surface of the substrates, although this technique is well known [28,29,32]. However, exposing the photoresist patterns to $\mathrm{O}_{2}$ plasma did not mitigate this problem, so it had to be resolved using surfactants.

Specifically, GOPS, a hydrolyzed silane crosslinker, was used to improve the anchoring, as its epoxy group can react open under acidic conditions, yielding a hydroxyl group. When GOPS is added to PEDOT:PSS suspensions, the hydroxyl groups strongly interact with the sulfonic acid groups of PEDOT:PSS via hydrogen bonds. The trimethoxysilane groups of GOPS are hydrolyzed in acidic suspensions and become $\mathrm{Si}-\mathrm{OH}$ groups, which form stable $\mathrm{Si}-\mathrm{O}-\mathrm{Si}$ bonds on the glass surface [32]. To reveal the effects of GOPS in the PEDOT:PSS suspensions, its concentration was varied, and the durability of the resulting PEDOT:PSS patterns was evaluated. As the GOPS concentration increased, fewer defects ended to appear in the PEDOT:PSS patterns. However, 3.0 wt \% GOPS caused PEDOT:PSS to agglomerate, which was previously reported to hinder spincoating [29]. Further, we confirmed that adding GOPS decreased the conductivity of the PEDOT:PSS films, suggesting a trade-off relationship between improving the durability and maintaining the conductivity of the PEDOT:PSS films. Figure 3 shows the relationship between the conductivity of PEDOT:PSS films and the GOPS concentration, which shows almost the same trend as that observed in previous work [28], although the initial conductivity without GOPS was slightly lower here. Based on these findings, we used 0.2 wt $\%$ GOPS in subsequent experiments because this concentration yielded PEDOT:PSS films that were sufficiently durable during the lift-off process. 
Surfaces, Interfaces and Coatings Technology
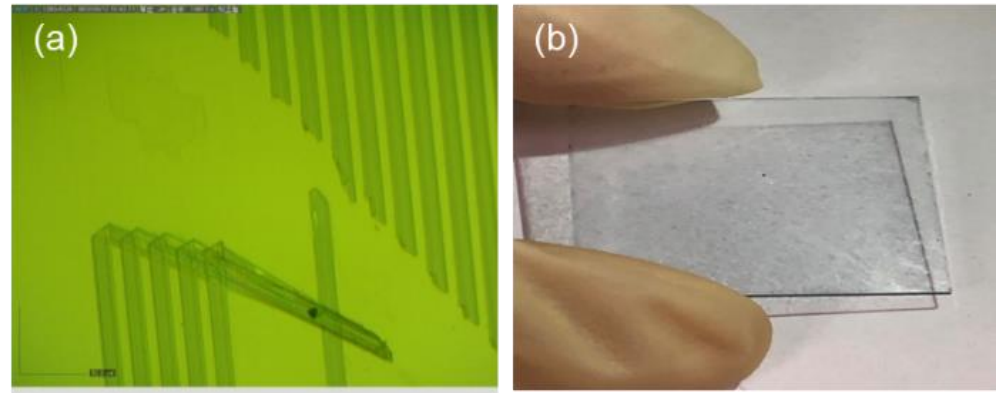

Figure 2: Poly(3,4-ethylenedioxythiophene) polystyrene sulfonate (PEDOT:PSS) film damaged when the substrate was shaken in an acetone bath during the lift-off process. (a) Image of the finger-type electrodes observed using an optical microscope. (b) Macroscale image of the entire substrate.

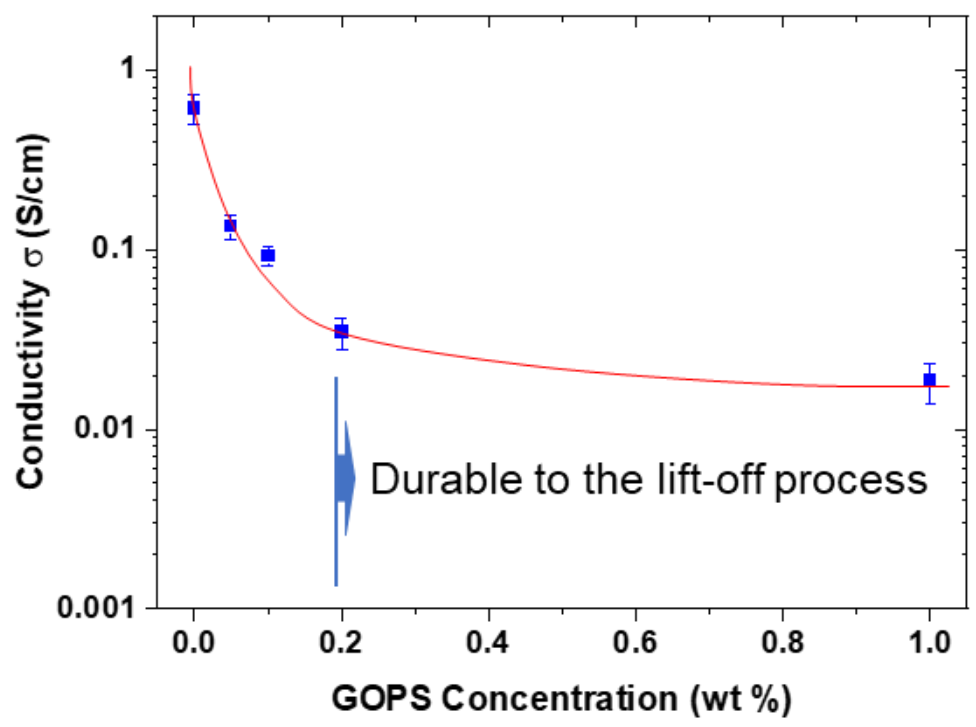

Figure 3: Changes in conductivity of PEDOT:PSS films when (3glycidyloxypropyl)trimethoxy-silane (GOPS) is added to PEDOT:PSS suspensions. The solid curve is a visual guide.

\section{Traditional Process for PEDOT:PSS}

Figure 4 shows the two-dimensional height profiles of the PEDOT:PSS patterns obtained using the traditional process. Each sample was measured at several points in the sample, and 
an average was taken at each position. Considering the photoresist patterns, we expect to observe a rectangular crosssection with a line width of $20 \mu \mathrm{m}$. Surprisingly, however, the actual profiles are U-shaped [33,34], with large variations in thickness at the center of the lines. The U-shaped profiles could be attributed to the evaporation of solvent and its corollary mass transfer [35]. If the surface of the photoresist has good wettability, the suspension spreads not only in the planar direction but also upward along the sidewalls of the photoresist. In addition, as the solvent evaporates, mass transfer occurs at the edge of the suspension on the sidewall of the photoresist, thereby leading to the U-shaped profiles at the edge. According to this mechanism, a higher concentration with higher viscosity would reduce the peak height of the U-shaped profiles, whereas better wettability, including on the sidewalls of the photoresist, would develop U-shaped profiles.

From another perspective, the bottom of the lines is approximately $30 \mu \mathrm{m}$, which is 1.5 times larger than the designed value. In contrast, the peak-to-peak width is almost 20 $\mu \mathrm{m}$, suggesting that each peak must be determined by the sidewall of the patterned photoresist. Presumably, when PEDOT:PSS is spin-coated onto the patterned photoresist, PEDOT:PSS not only fills the spaces between the lines of the patterned photoresist but also covers the top of the photoresist. The PEDOT:PSS initially present on top of the photoresist can be connected to the PEDOT:PSS between the lines via conformal coverage. Therefore, even after the photoresist patterns are removed during the lift-off process, partially connected PEDOT:PSS would remain at the bottom of the patterns [30]. These observations suggest that removing the PEDOT:PSS from the top of the photoresist may help improve the quality and uniformity of the final PEDOT:PSS patterns. 


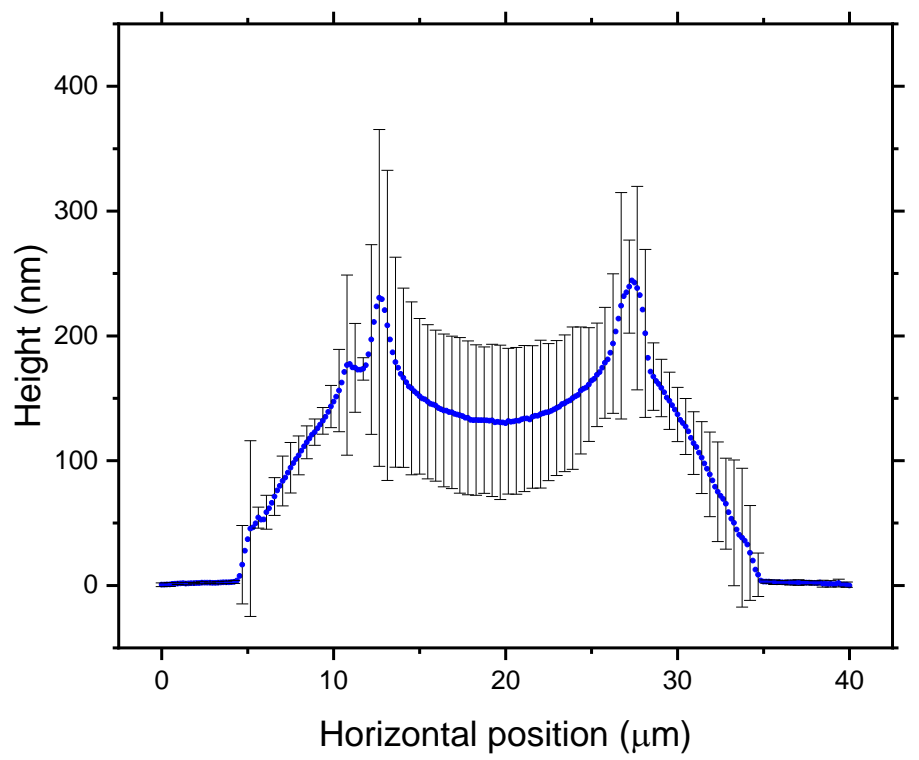

Figure 4: Height profiles of the patterned PEDOT:PSS lines made using the traditional process. The error bars show the difference in the maximum and minimum values at each position.

\section{Adding an $\mathrm{O}_{2}$ Plasma Treatment before Spin-Coating}

$\mathrm{O}_{2}$ plasma increases the hydrophilicity of surfaces. Contact angle measurements revealed that the surface of the patterned photoresist was rather hydrophobic, which prompted us to add an $\mathrm{O}_{2}$ plasma treatment before spin-coating PEDOT:PSS, which would also remove the PEDOT:PSS from the top of the patterned photoresist. Figure 5 shows how PEDOT:PSS suspensions spread on the surface of the photoresist with and without an $\mathrm{O}_{2}$ plasma treatment; clearly, increasing the $\mathrm{O}_{2}$ plasma operation time further increased the wettability of the patterned photoresist surface. 


\section{Surfaces, Interfaces and Coatings Technology}

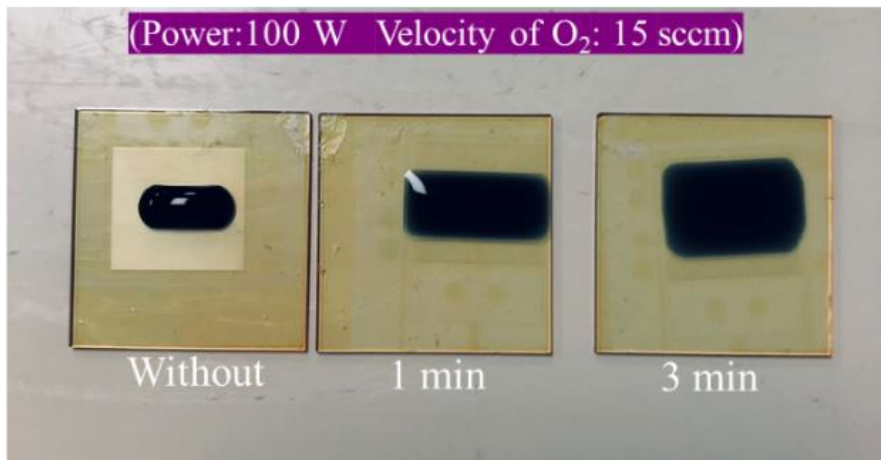

Figure 5: Effects of exposing $\mathrm{O}_{2}$ plasma to the surface of the patterned photoresist on its wettability.
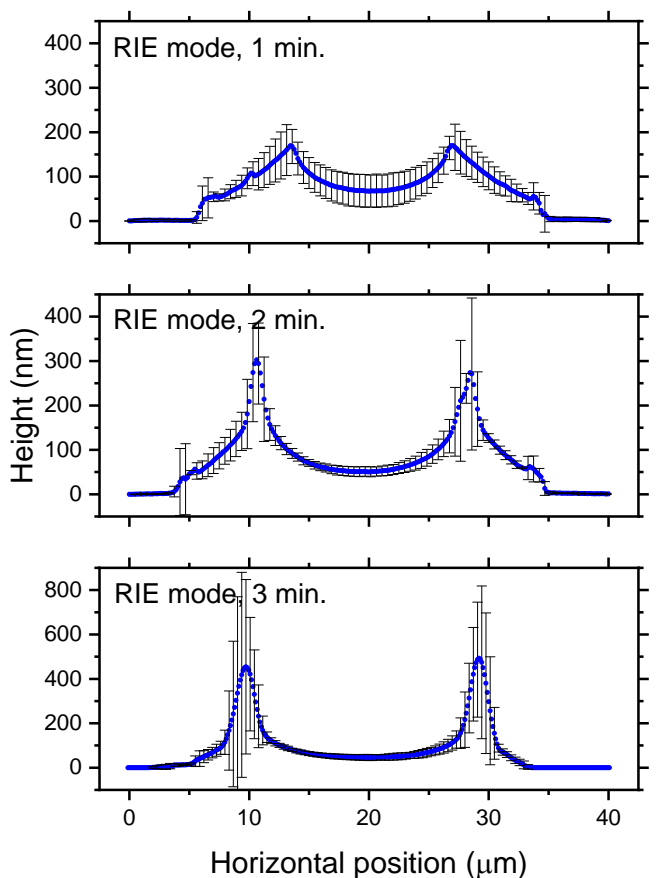

Figure 6: Height profiles of the patterned PEDOT:PSS lines made by plasmatreating the photoresist in reaction ion etching (RIE) mode before spin-coating PEDOT:PSS. The error bars show the maximum and minimum values at each position. 
We found that adding the $\mathrm{O}_{2}$ plasma treatment at this point during processing indeed improves the uniformity of the thickness. Figure 6 shows how the height profiles of the crosssectional PEDOT:PSS patterns change when the patterned photoresist is plasma-treated in RIE mode before spin-coating it with PEDOT:PSS. As the operating time of the $\mathrm{O}_{2}$ plasma treatment increases, the center of the lines becomes more uniform. Interestingly, however, the peaks that appear at the edge of each line become more pronounced with the increasing operating time, which suggests that the plasma-treated surface of the photoresist-patterned substrates indeed provides better wettability for PEDOT:PSS and hence better uniformity at the center of the lines. Specifically, the thickness variations at the center of the lines are improved, decreasing from \pm 33.9 to \pm 5.7 $\mathrm{nm}$. Furthermore, $\mathrm{O}_{2}$ plasma increases the wettability of the sidewalls of the photoresist patterns, and therefore, the peaks become more pronounced with the increasing operating time owing to the so-called coffee ring effect $[35,36]$. This finding can be verified by using a gentler $\mathrm{O}_{2}$ plasma treatment in $\mathrm{PE}$ mode.

Figure 7 shows how the height profiles of the cross-sectional PEDOT:PSS patterns change when the photoresist is plasmatreated in PE mode before spin-coating PEDOT:PSS. In contrast to the results of the RIE $\mathrm{O}_{2}$ plasma treatment, the thickness of PEDOT:PSS changes more mildly and gently, with little impact on the sidewalls of the photoresist pattern. Figure 8 compares the changes in thickness and its variability at the center of the PEDOT:PSS patterns when the patterned photoresist is plasmatreated in RIE or PE mode before spin-coating. In RIE mode, the $\mathrm{O}_{2}$ plasma treatment is indeed vigorous and more rapidly reduces the film thickness. Presumably, this mode makes the surface of the photoresist more hydrophilic than PE mode, which is consistent with the results shown in Figure 5. Although in principle, the RIE plasma mode bombards the substrate surface more vertically, it also more severely affects the sidewalls. 

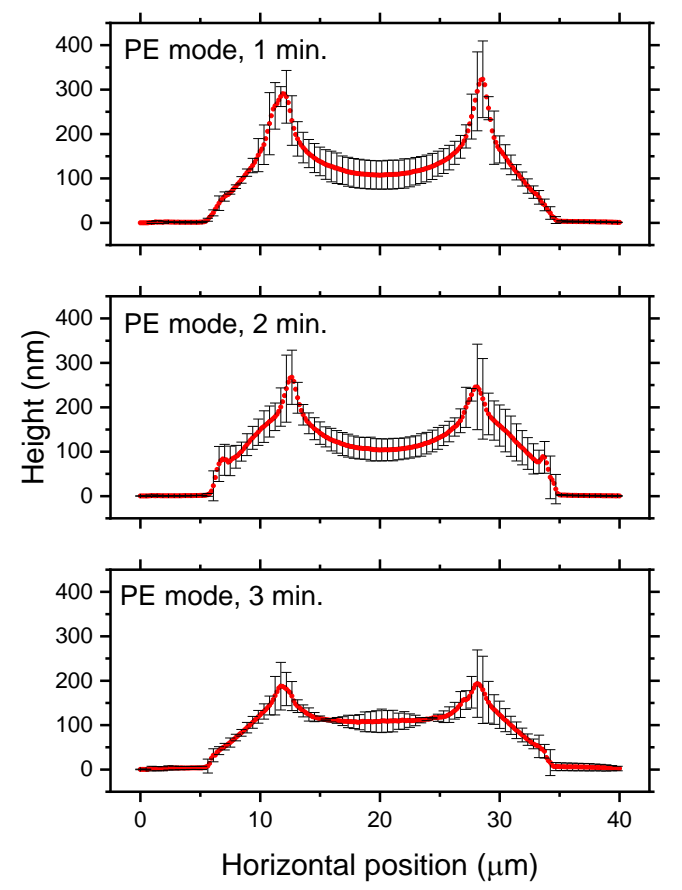

Figure 7: Height profiles of the patterned PEDOT:PSS lines made by plasmatreating the photoresist in plasma etching (PE) mode before spin-coating PEDOT:PSS. The error bars show the maximum and minimum values at each position.

Therefore, the hydrophilicity increases on the entire surface of the patterned photoresist, including the sidewalls, so the Ushaped profiles become more pronounced. Meanwhile, the films become thinner, with less variability at the center of each patterned line and a higher peak at the edge.

In subsequent experiments, to avoid developing U-shaped crosssectional profiles in the PEDOT:PSS patterns, we performed the $\mathrm{O}_{2}$ plasma treatment for $3 \mathrm{~min}$ in PE mode before spin-coating PEDOT:PSS for the following step, i.e., lifting off the photoresist. 
Surfaces, Interfaces and Coatings Technology

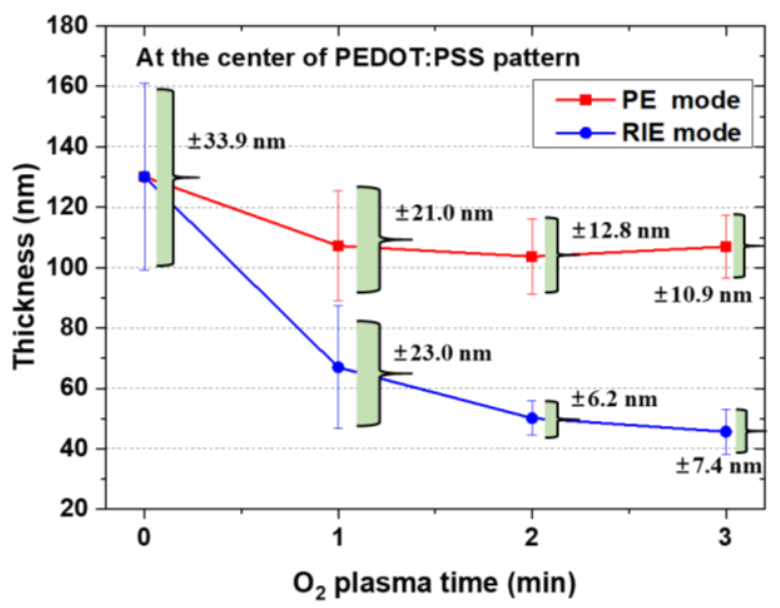

Figure 8: Changes in thickness and its variability at the center of the PEDOT:PSS patterns as a function of $\mathrm{O}_{2}$ plasma exposure time using PE and RIE modes.

\section{Adding an $\mathrm{O}_{2}$ Plasma Treatment before Lifting Off the Photoresist}

The next step introduces another difficulty that disturbs the uniformity of the patterns. Undesirable patterns, a type of defect, are often observed when lifting off the photoresist. Figure 9 shows an image of typical undesirable patterns remaining on the surface of the patterned PEDOT:PSS, which we assume are related to the PEDOT:PSS covering the top of the photoresist via conformal coverage [30]. Presumably, when the photoresist patterns are lifted off, the PEDOT:PSS anchored to the surface of the photoresist mechanically causes this problem. Further, brush-like wrinkles often appear around the boundaries after spin-coating PEDOT:PSS onto the patterned photoresist, as shown in Figure 10b, which prompts us to consider the effects of conformal coverage. During the spin-coating process, PEDOT:PSS spreads over the entire patterned photoresist surface, meaning that PEDOT:PSS can spread not only in the gaps between the strip patterns but also on top of the patterned lines. Therefore, removing such PEDOT:PSS covering the photoresist before lifting it off would help reduce these defects. 
Surfaces, Interfaces and Coatings Technology

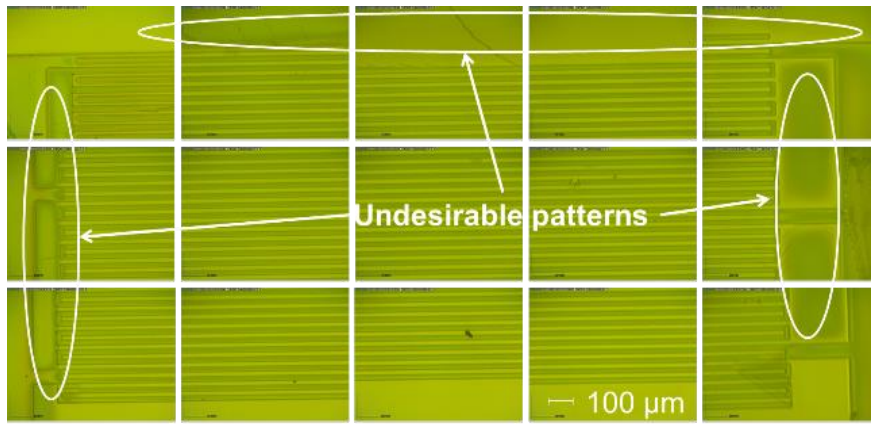

Figure 9: Typical undesirable patterns remaining on the surface of the patterned PEDOT:PSS observed using an optical microscope.
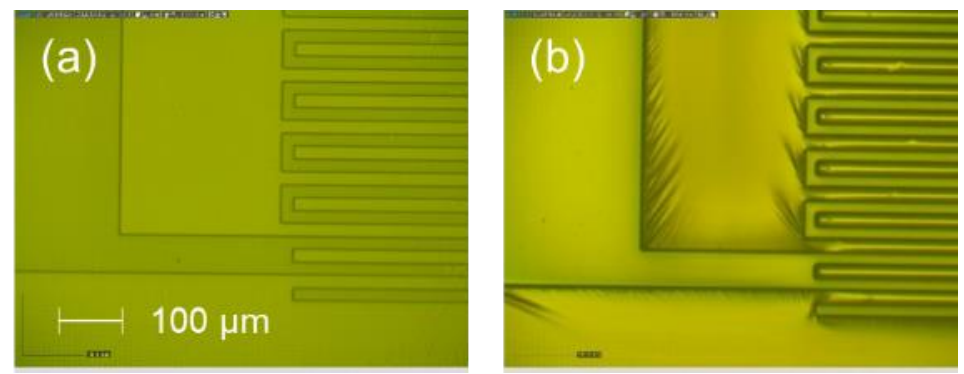

Figure 10: Photoresist patterns (a) before and (b) after spin-coating PEDOT:PSS observed using an optical microscope.

Thus, to remove the PEDOT:PSS from the top of the photoresist caused by conformal coverage, we introduce another $\mathrm{O}_{2}$ plasma process before lifting off the photoresist patterns. As expected, applying this additional $\mathrm{O}_{2}$ plasma treatment at this stage reduces the undesirable patterns and defects in the resulting PEDOT:PSS when the photoresist is finally removed with acetone, as shown in Figure 11. Obviously, however, a longer plasma treatment at this stage also reduces the thickness of PEDOT:PSS itself or even damages the patterns. As an extreme case in which we plasma-treated the PEDOT:PSS pattern for $120 \mathrm{~s}$, its thickness decreased to one-fifth of its designed value, and its linewidths became two-thirds narrower. Further, we observed jagged line patterns, which are side effects of $\mathrm{O}_{2}$ plasma exposure at this stage. Therefore, the operating time of $\mathrm{O}_{2}$ plasma has a trade-off 
relationship between mitigating these undesirable patterns and maintaining the quality of the PEDOT:PSS patterns.

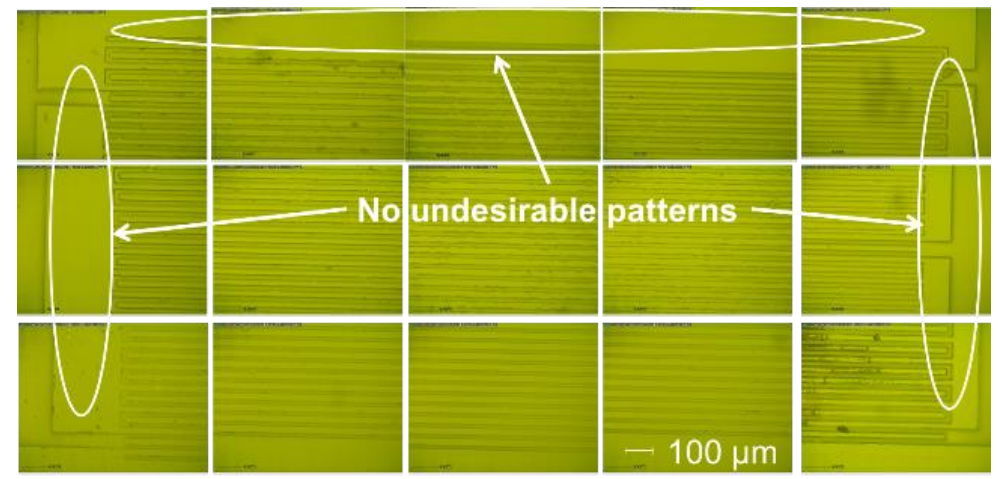

Figure 11: Microscopic image of undesirable patterns being removed when adding $\mathrm{O}_{2}$ plasma in RIE mode for $15 \mathrm{~s}$.

Nevertheless, adding an $\mathrm{O}_{2}$ plasma treatment at this stage enables the removal of the unfavorable PEDOT:PSS covering the photoresist, suggesting that it is crucial to remove such PEDOT:PSS from the top of the photoresist or avoid conformal coverage. In order to remove this PEDOT:PSS without affecting the film thickness or damaging the film, $\mathrm{O}_{2}$ plasma etching with masking would be more reliable.

\section{Combination of the Added $\mathrm{O}_{2}$ Plasma Treatments}

Naturally, we are also interested in how combining the two added $\mathrm{O}_{2}$ plasma processes eventually improves the quality of the PEDOT:PSS patterns. We thus optimized the plasma treatment operation time in PE mode to be 3 min before spincoating PEDOT:PSS and that before lifting off the photoresist in RIE mode to be $1 \mathrm{~s}$. Our investigation reveals that even $1 \mathrm{~s}$ of $\mathrm{O}_{2}$ plasma in the RIE mode assists with removing undesirable PEDOT:PSS, thereby promoting the lift-off process while avoiding defects. Figure 12 shows microscopic images and height profiles of the PEDOT:PSS patterns when both $\mathrm{O}_{2}$ plasma treatments are added to the process. Applying both treatments enables fabricating more uniform, defect-free PEDOT:PSS finger-type patterns with a deviation in the thickness of $\pm 10.6 \mathrm{~nm}$ 
at the center of the cross-section. The bottom of the lines is still beyond the designed value, thus requiring further optimization; however, avoiding conformal coverage would be crucial to improving microfabrication using photolithography.
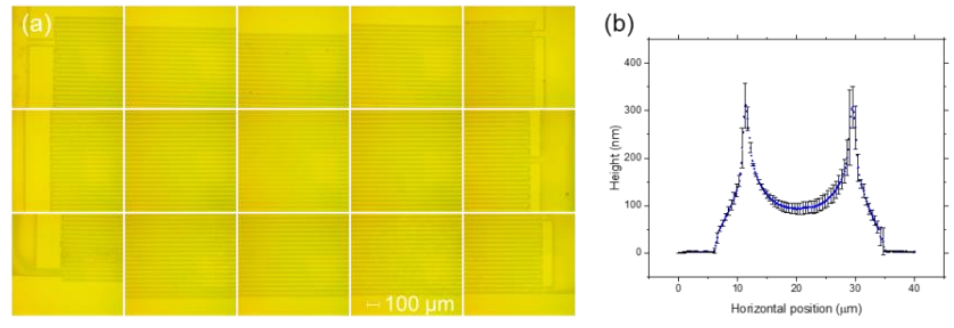

Figure 12: (a) Microscopic images and (b) height profiles of the PEDOT:PSS patterns made by adding both $\mathrm{O}_{2}$ plasma treatments to traditional photolithography.

\section{Conclusions}

We fabricated finger-type PEDOT:PSS electrodes using conventional photolithographic patterning techniques, during which the cross-sectional U-shaped profiles of the PEDOT:PSS patterns were found instead of rectangular cross-sections. The fragility of the PEDOT:PSS patterns was improved by adding 0.2 wt \% GOPS to the PEDOT:PSS suspensions. Using the strengthened PEDOT:PSS patterns, we investigated the effects of adding $\mathrm{O}_{2}$ plasma treatments to the traditional photolithography process for patterning PEDOT:PSS, expecting to observe how this treatment improved the uniformity of the patterns and how it changed the thickness and U-shaped profiles of the patterns. One $\mathrm{O}_{2}$ plasma treatment was introduced before spin-coating PEDOT:PSS onto the patterned photoresist layer, and the other was employed before the lift-off process for removing the photoresist layer. The former improved the wettability of the patterned photoresist surface, including that of its sidewalls, and the latter helped partially remove the spin-coated PEDOT:PSS that impeded the lift-off process. In the former $\mathrm{O}_{2}$ plasma treatment, the uniformity of the thickness at the center of the lines became more uniform, with an improved deviation from several tens of nanometers to several nanometers; however, the 
U-shaped patterns became more pronounced when the sidewalls of the patterned photoresist were exposed to the plasma in RIE mode, which could be avoided using more gentle plasma in PE mode. The latter $\mathrm{O}_{2}$ plasma treatment eventually facilitated the lift-off process while preventing defects, uniformly reducing the entire thickness of the PEDOT:PSS patterns while removing the PEDOT:PSS covering the photoresist. Our findings suggest that the most important factor is how to remove PEDOT:PSS from the top of the photoresist or avoid its conformal coverage. Applying these two additional processes enabled fabricating more uniform, defect-free PEDOT:PSS patterns. Realizing a real $\mathrm{THz}$ device using the investigated techniques requires considerable effort to optimize the entire procedure, which would involve many factors, such as the cross-sectional shape of the patterned lines, defects, conductivity, and anchoring strength.

\section{References}

1. Zhang $\mathrm{XC}, \mathrm{Xu}$ J. Introduction to $\mathrm{THz}$ Wave Photonics. New York: Springer US. 2010.

2. Shi JW, Huang CB, Pan CL. Millimeter-wave photonic wireless links for very high data rate communication. NPG Asia Mater. 2011; 3: 41-48.

3. Chen CY, Hsieh CF, Lin YF, Pan RP, Pan CL. Magnetically tunable room-temperature $2 \pi$ liquid crystal terahertz phase shifter. Opt. Express. 2004; 12: 2625-2630.

4. Wu HY, Hsieh CF, Tang TT, Pan RP, Pan CL. Electrically tunable room-temperature $2 \pi$ liquid crystal terahertz phase shifter. IEEE Photon. Technol. Lett. 2006; 18: 1488-1490.

5. Lin $\mathrm{Xw}, \mathrm{Wu} \mathrm{Jb}, \mathrm{Hu} \mathrm{W}$, Zheng $\mathrm{Zg}, \mathrm{Wu} \mathrm{Zj}$, et al. Selfpolarizing terahertz liquid crystal phase shifter. AIP Adv. 2011; 1: 032133.

6. Yang CS, Tang TT, Chen PH, Pan RP, Yu P, et al. Voltagecontrolled liquid-crystal terahertz phase shifter with indiumtin-oxide nanowhiskers as transparent electrodes. Opt. Lett. 2014; 39: 2511.

7. Yang CS, Tang TT, Pan RP, Yu P, Pan CL. Liquid crystal terahertz phase shifters with functional indium-tin-oxide nanostructures for biasing and alignment. Appl. Phys. Lett. 2014; 104: 141106.

8. Yang CS, Kuo C, Chen PH, Wu WT, Pan RP, et al. High- 
transmittance $2 \pi$ electrically tunable terahertz phase shifter with CMOS-compatible driving voltage enabled by liquid crystals. Appl. Sci. 2019; 9: 271.

9. Chiang YJ, Yang CS, Yang YH, Pan CL, Yen TJ. An ultrabroad terahertz bandpass filter based on multipleresonance excitation of a composite metamaterial. Appl. Phys. Lett. 2011; 99: 191909.

10. Chatzakis I, Tassin P, Luo L, Shen NH, Zhang L, et al. Oneand two-dimensional photo-imprinted diffraction gratings for manipulating terahertz waves. Appl. Phys. Lett. 2013; 103: 043101.

11. Hsieh CF, Lai YC, Pan RP, Pan CL. Polarizing terahertz waves with nematic liquid crystals. Opt. Lett. 2008; 33: 1174.

12. Sahoo AK, Yang CS, Wada O, Pan CL. Twisted nematic liquid crystal based terahertz phase shifter with crossed indium tin oxide finger type electrodes. IEEE Trans. Terahertz Sci. Technol. 2019; 9: 399-408.

13. Yang CS, Kuo C, Tang CC, Chen JC, Pan RP, et al. Liquidcrystal terahertz quarter-wave plate using chemical-vapordeposited graphene electrodes. IEEE Photon. J. 2015; 7: 2200808.

14. Kim N, Kee S, Lee SH, Lee BH, Kahng YH, et al. . Highly conductive PEDOT:PSS nanofibrils induced by solutionprocessed crystallization. Adv. Mater. 2014; 26: 2268-2272.

15. Sun K, Zhang S, Li P, Xia Y, Zhang X, et al. Review on application of PEDOTs and PEDOT:PSS in energy conversion and storage devices. J. Mater. Sci. Mater. Electron. 2015; 26: 4438-4462.

16. Ouyang S, Xie Y, Wang D, Zhu D, Xu X, et al. Surface patterning of PEDOT:PSS by photolithography for organic electronic devices. J. Nanomater. 2015; 2015: 603148.

17. Sun Y, Yang S, Du P, Yan F, Qu J, et al. Investigate the effects of EG doping PEDOT/PSS on transmission and antireflection properties using terahertz pulsed spectroscopy. Opt. Express. 2017; 25: 1723.

18. Rwei SP, Lee YH, Shiu JW, Sasikumar R, Shyr UT. Characterization of solvent-treated PEDOT:PSS thin films with enhanced conductivities. Polymers. 2019; 11: 134.

19. Huseynova G, Hyun Kim Y, Lee JH, Lee J. Rising advancements in the application of PEDOT:PSS as a prosperous transparent and flexible electrode material for solution-processed organic electronics. J. Inf. Disp. 2020; 
Surfaces, Interfaces and Coatings Technology

21:71-91.

20. Ling H, Liu S, Zheng Z, Yan F. Organic flexible electronics. Small Methods. 2018; 2: 1800070.

21. Fan X, Nie W, Tsai $H$, Wang $N$, Huang $H$, et al. PEDOT:PSS for flexible and stretchable electronics: Modifications, strategies, and applications. Adv. Sci. 2019; 6: 1900813.

22. Hwang HS, Zakhidov AA, Lee JK, André X, Defranco JA, et al. Dry photolithographic patterning process for organic electronic devices using supercritical carbon dioxide as a solvent. J. Mater. Chem. 2008; 18: 3087-3090.

23. Lee JK, Taylor PG, Zakhidov AA, Fong HH, Hwang HS, et al. Orthogonal processing: A novel photolithographic patterning method for organic electronics. J. Photopolym. Sci. Technol. 2009; 22: 565-569.

24. Khong SH, Sivaramakrishnan S, Png RQ, Wong LY, Chia PJ, et al. General photo-patterning of polyelectrolyte thin films via efficient ionic bis(fluorinated phenyl azide) photocrosslinkers and their post-deposition modification. Adv. Funct. Mater. 2007; 17: 2490-2499.

25. Winroth G, Latini G, Credgington D, Wong LY, Chua LL, et al. Polyfluorene-based light-emitting diodes with an azide photocross-linked poly(3,4-ethylene dioxythiophene):(polystyrene sulfonic acid) hole-injecting layer. Appl. Phys. Lett. 2008; 92: 103308.

26. Fenwick O, Oliver K, Cacialli F. Cross-linking of a poly(3,4ethylene dioxythiophene):(polystyrene sulfonic acid) hole injection layer with a bis-azide salt and the effect of atmospheric processing conditions on device properties. Appl. Phys. Lett. 2012; 100: 053309.

27. Yoshida S, Hagiwara K, Hasebe T, Hotta A. Surface modification of polymers by plasma treatments for the enhancement of biocompatibility and controlled drug release. Surf. Coatings Technol. 2013; 233: 99-107.

28. Håkansson A, Han S, Wang S, Lu J, Braun S, et al. Effect of (3-glycidyloxypropyl)trimethoxysilane (GOPS) on the electrical properties of PEDOT:PSS films. J. Polym. Sci. Part B Polym. Phys. 2017; 55: 814-820.

29. He J, Wan Y, Gao P, Tang J, Ye J. Over $16.7 \%$ efficiency organic-silicon heterojunction solar cells with solutionprocessed dopant-free contacts for both polarities. Adv. Funct. Mater. 2018; 28: 1802192.

30. Chan JR, Huang XQ, Song AM. Nondestructive 
Surfaces, Interfaces and Coatings Technology

photolithography of conducting polymer structures. J. Appl. Phys. 2006; 99: 023710.

31. Athreya S, Venkatesh YD. Application of Taguchi method for optimization of process parameters in improving the surface roughness of lathe facing operation. Int. Ref. J. Eng. Sci. 2012; 1: 13-19.

32. Yoon SS, Khang DY. Roles of nonionic surfactant additives in PEDOT:PSS thin films. J. Phys. Chem. C 2016; 120: 29525-29532.

33. Wang X, Östblom M, Johansson T, Inganäs O. PEDOT surface energy pattern controls fluorescent polymer deposition by dewetting. Thin Solid Films. 2004; 449: 125132.

34. Charlot B, Sassine G, Garraud A, Sorli B, Giani A, et al. Micropatterning PEDOT:PSS layers. Microsyst. Technol. 2013; 19: 895-903.

35. Deegan RD, Bakajin O, Dupont TF, Huber G, Nagel SR, et al. Capillary flow as the cause of ring stains from dried liquid drops. Nature. 1997; 389: 827-829.

36. Deegan RD, Bakajin O, Dupont TF, Huber G, Nagel SR, et al. Contact line deposits in an evaporating drop. Phys. Rev. E Stat. Phys. Plasmas Fluids Relat. Interdiscip. Top. 2000; 62: 756-765. 